\title{
Survey of eriophyid mites on some fruit trees, with re-descriptions of two newly recorded species and a checklist of eriophyid mites in Egypt (Acari: Eriophyoidea)
}

\author{
Ashraf S. H. El-Halawany \\ E-mail dr ashraf said@yahoo.com \\ Plant Protection Research Institute (PPRI), Agricultural Research Center (ARC), \\ Dokki, Giza, 12618 Egypt
}

\begin{abstract}
In a field survey, 16 eriophyoid mite species were collected from eight species of fruit trees, among which two species were found to be new record to Egypt. They were Tegolophus guavae (Boczek, 1960) on Psidium guajava L. (Myrtaceae) which causes rust on leaves, and Aceria ziziphi Mohanasundaram, 1990, vagrant without damage on Ziziphus spina-christi Willd (Rhamnaceae). These species were redescribed and illustrated from local material. A total of 65 eriophyoid mite species in superfamily Eriophyoidea has been recorded in Egypt due to literature. These species belong to three families, seven sub families, seven tribes and 27 genera. A list of these species has been constructed and presented herein.
\end{abstract}

Key words: Survey, Eriophyidae, Phytoptidae, Diptilomiopidae, Checklist, Egypt.

\section{INTRODUCTION}

Eriophyid mites play an important role in Egyptian agro-ecosystem. The last collective work on eriophyid mites' taxonomy was occurred by Zaher (1984). About 30 papers on taxonomy of eriophyid mites in Egypt, from 1919 up to date, have been published by (Dçbski, 1919; Hassan, 1934; Sayed, 1946a, 1946b; Attiah, 1955, 1967 \& 1970; Osman \& Zohdy, 1976; Soliman \& Abou-Awad, 1977 \& 1978; Rasmy \& Abou-Awad, 1978; Abou-Awad, 1979a, 1979b, 1981 \& 1984; Abou-Awad \& Nasr, 1983a, 1983b \& 1986; Zaher, 1984; Zaher \& Abou-Awad, 1978, 1979, 1980a \& 1980b; Zaher et al., 1978; Osman \& Abou-Taka, 1989; Abou-Awad \& El-Sawi, 1993; Abou-Awad \& El-Borolossy, 1995; El-Halawany et al., 2001 and Abou Awad et al., 2011. About 63 species were recorded. The presented work aimed to survey eriophyid mites on eight fruit trees and to collect the information in tables, related to their host plants. In addition, redescriptions of two species recorded for the first time in Egypt are presented. A checklist to the Egyptian eriophyoid mites are provided too.

\section{MATERIAL AND METHODS}

A survey was carried out in Qalubia Governorate included eight species of fruit trees, namely Guava (Psidium guajava L.), Christ thorn (Ziziphus spina-christi Willd), Date palm (Phoenix dactylifera L.), Apple (Malus domestica Borkh.), Sycamore fig (Ficus sycomorus L.), Olive (Olea europaea L.), Fig (Ficus carica L.) and Mulberry (Morus alba L.). Sampling was carried out irregularly from October 2011 to September 2012 including plant foliages, branches, flowers and buds. Samples were individually bagged in tightly-closed plastic bags and transported the same day to the Fruit Acarology Department, Plant Protection Research Institute 
(PPRI), Agricultural Research Center (ARC) for mite extraction. A microscope with an attached drawing tube was used for examination and drawing the mite species Tegolophus guavae and Aceria ziziphi which were recorded for the first time in Egypt. The oil lens was used to examine the fetherclaw, microtubercles and male and female genitalia. The measurements are given in micrometer $(\mu \mathrm{m})$ and GPS technique is used to identify located samples. The terminology and morphological characteristics used in this study are based on Lindquist (1996) and Amrine et al. (2003). All specimens were deposited in the Fruit Acarology Department, Plant Protection Research Institute collection, Agricultural Research Center.

More than 28 original scientific papers published on eriophyid mites from Egypt were collected from different libraries in Egyptian Universities and Research Centers. On the other side, the references collected from Egypt were confirmed by the Catalogue of the Eriophyoidea of the world (Version computer of Filemaker Pro 4.0. (De Lillo \& Amrine, 2009) personal communication). All eriophyid species recorded in Egypt and their host plants are now up to date.

\section{RESULTS AND DISCUSSION}

\section{Survey of eriophyid mites on eight fruit crops}

Sixteen eriophyid mite species were collected from eight fruit trees species at Qalubia Governorate. The host plant and GPS are provided (Table 1).

Table 1: Results of survey of eriophyoid mites from Qalubia Governorate.

\begin{tabular}{|c|c|c|c|}
\hline Classification & Scientific name & Host plant & $\overline{\text { GPS }}$ \\
\hline $\begin{array}{l}\text { Family: Diptilomiopidae } \\
\text { Sub family: Diptilomiopinae }\end{array}$ & Diptilomiopus ficus Attiah, 1967 & $\begin{array}{l}\text { Ficus carica L., } \\
\text { (Moraceae) }\end{array}$ & $\begin{array}{l}30^{\circ} 20^{\prime} 18.67^{\prime \prime} \mathrm{N} \\
31^{\circ} 15^{\prime} 15.12^{\prime \prime} \mathrm{E}\end{array}$ \\
\hline Sub family: Rhyncaphytoptinae & Rhyncaphytoptus ficifoliae Keifer, 1939a & $\begin{array}{l}\text { Ficus carica L., } \\
\text { (Moraceae) }\end{array}$ & $\begin{array}{l}30^{\circ} 20^{\prime} 18.67^{\prime \prime} \mathrm{N} \\
31^{\circ} 15^{\prime} 15.12^{\prime \prime} \mathrm{E}\end{array}$ \\
\hline \multirow{6}{*}{$\begin{array}{l}\text { Family: Eriophyidae } \\
\text { Sub family: Eriophyinae } \\
\text { Tribe: Aceriini }\end{array}$} & Aceria ficus (Cotté, 1920) & $\begin{array}{l}\text { Ficus carica L., } \\
\text { (Moraceae) }\end{array}$ & $\begin{array}{l}30^{\circ} 20^{\prime} 18.67^{\prime \prime} \mathrm{N} \\
31^{\circ} 15^{\prime} 15.12^{\prime \prime} \mathrm{E}\end{array}$ \\
\hline & Aceria mori (Keifer, 1939a) & $\begin{array}{l}\text { Morus alba L., } \\
\text { (Moraceae) }\end{array}$ & $\begin{array}{l}30^{\circ} 16^{\prime} 10.30^{\prime \prime} \mathrm{N} \\
31^{\circ} 14^{\prime} 37.88^{\prime \prime} \mathrm{E}\end{array}$ \\
\hline & Aceria oleae (Nalepa, 1900) & $\begin{array}{l}\text { Olea europaea L., } \\
\text { (Oleaceae) }\end{array}$ & $\begin{array}{c}30^{\circ} 1^{\prime} 8.21^{\prime \prime} \mathrm{N} \\
31^{\circ} 12 ' 28.04^{\prime \prime} \mathrm{E}\end{array}$ \\
\hline & Aceria olivi (Zaher \& Abou-Awad, 1979) & $\begin{array}{l}\text { Olea europaea L., } \\
\text { (Oleaceae) }\end{array}$ & $\begin{array}{c}30^{\circ} 1^{\prime} 8.21^{\prime \prime} \mathrm{N} \\
31^{\circ} 12 ' 28.04 " \mathrm{E}\end{array}$ \\
\hline & $\begin{array}{l}\text { Aceria sycamori (Soliman \& Abou-Awad, } \\
\text { 1977) }\end{array}$ & $\begin{array}{l}\text { Ficus sycomorus L., } \\
\text { (Moraceae) }\end{array}$ & $\begin{array}{l}30^{\circ} 18^{\prime} 37.17^{\prime \prime} \mathrm{N} \\
31^{\circ} 15^{\prime} 49.27^{\prime \prime} \mathrm{E}\end{array}$ \\
\hline & $\begin{array}{l}\text { Aceria ziziphi Mohanasundaram, 1990; } \\
\text { New record }\end{array}$ & $\begin{array}{l}\text { Ziziphus spina-christi } \\
\text { Willd (Rhamnaceae) }\end{array}$ & $\begin{array}{l}30^{\circ} 15^{\prime} 50.46^{\prime \prime} \mathrm{N} \\
31^{\circ} 14^{\prime} 51.85^{\prime \prime} \mathrm{E}\end{array}$ \\
\hline Tribe: Eriophyini & Eriophyes pyri (Pagenstecher, 1857) & $\begin{array}{l}\text { Malus domestica Borkh., } \\
\text { (Rosaceae) }\end{array}$ & $\begin{array}{c}30^{\circ} 10^{\prime} 59.89^{\prime \prime} \mathrm{N} \\
31^{\circ} 7{ }^{\prime} 39.52^{\prime \prime} \mathrm{E}\end{array}$ \\
\hline \multirow[t]{3}{*}{$\begin{array}{l}\text { Sub family: Phyllocoptinae } \\
\text { Tribe: Anthocoptini }\end{array}$} & Tegolophus hassani (Keifer, 1959) & $\begin{array}{l}\text { Olea europaea L., } \\
\text { (Oleaceae) }\end{array}$ & $\begin{array}{c}30^{\circ} 1^{\prime} 8.21^{\prime \prime} \mathrm{N} \\
31^{\circ} 12 ' 28.04^{\prime \prime} \mathrm{E}\end{array}$ \\
\hline & $\begin{array}{l}\text { Tegolophus guavae (Boczek, 1960); New } \\
\text { record }\end{array}$ & $\begin{array}{l}\text { Psidium guajava L. } \\
\text { (Myrtaceae) }\end{array}$ & $\begin{array}{l}30^{\circ} 14^{\prime} 51.85^{\prime \prime} \mathrm{N} \\
31^{\circ} 17^{\prime} 07.89^{\prime \prime} \mathrm{E} \\
\end{array}$ \\
\hline & Tegolophus niloticus Abou-Awad, 1984 & $\begin{array}{l}\text { Ficus sycomorus L., } \\
\text { (Moraceae) }\end{array}$ & $\begin{array}{l}30^{\circ} 18^{\prime} 37.17^{\prime \prime N} \\
31^{\circ} 15^{\prime} 49.27^{\prime \prime} \mathrm{E} \\
\end{array}$ \\
\hline \multirow[t]{2}{*}{ Tribe: Phyllocoptini } & Calepitrimerus baileyi Keifer (1938b) & $\begin{array}{l}\text { Malus domestica Borkh., } \\
\text { (Rosaceae) }\end{array}$ & $\begin{array}{c}30^{\circ} 10^{\prime} 59.89^{\prime \prime} \mathrm{N} \\
31^{\circ} 7^{\prime} 39.52^{\prime \prime} \mathrm{E}\end{array}$ \\
\hline & Epitrimerus pyri (Nalepa, 1891) & $\begin{array}{l}\text { Malus domestica Borkh., } \\
\text { (Rosaceae) }\end{array}$ & $\begin{array}{c}30^{\circ} 10^{\prime} 59.89^{\prime \prime} \mathrm{N} \\
31^{\circ} 7^{\prime} 39.52^{\prime \prime} \mathrm{E}\end{array}$ \\
\hline Tribe: Tegonotini & Oxycenus maxwelli (Keifer, 1939a) & $\begin{array}{l}\text { Olea europaea L., } \\
\text { (Oleaceae) }\end{array}$ & $\begin{array}{c}30^{\circ} 1^{\prime} 8.21^{\prime \prime} \mathrm{N} \\
31^{\circ} 12 ' 28.04 " \mathrm{E}\end{array}$ \\
\hline \begin{tabular}{|l} 
Subfamily: Sierraphytoptinae \\
Tribe: Mackiellini
\end{tabular} & Mackiella phoenicis Keifer, 1939a & $\begin{array}{l}\text { Phoenix dactylifera L., } \\
\text { (Arecaceae ) }\end{array}$ & $\begin{array}{l}30^{\circ} 17^{\prime} 20.02^{\prime \prime} \mathrm{N} \\
31^{\circ} 14^{\prime} 51.85^{\prime \prime} \mathrm{E} \\
\end{array}$ \\
\hline
\end{tabular}




\section{Re-descriptions of two newly recorded species}

Two species Tegolophus guavae (Boczek) and Aceria ziziphi Mohanasundram were recorded on Psidium guajava and Ziziphus spina-christi for the first time in Egypt. The following are re-descriptions of them.

Tegolophus guavae (Boczek, 1960) (Fig. 1)

Tegonotus guavae Boczek, 1960: 11.

Tegolophus guavae (Boczek, 1960) Amrine \& Stasny, 1996: 300.

Material examined: Qalubia; 6.VIII.2012, (Ashraf El-Halawany), Psidium guajava L., $(10$ 우, 6 ○ð $)\left(30^{\circ} 14^{\prime} 57.94^{\prime \prime} \mathrm{N} ; 31^{\circ} 17^{\prime} 07.89^{\prime \prime} \mathrm{E}\right)$.

FEMALE: Body fusiform, light yellowish in colour, 204 (180-225) long, 72 (60-80) wide; Gnathosoma16 (15-17), projecting obliquely downwards, pedipalp coxal setae (ep) 2 (2-3), dorsal pedipalp genual setae (d) 4 (4-5), chelicerae 14.5 (13.5-15.5), oral stylets 14 (12-16). Prodorsal shield rounded shape in rear with 39.5 (35-46) long, 55.5 (46-70) wide; median line split near center of shield into two lines which do not reach anterior margin, surface of shield punctuated. Frontal lobe broad 5.5 (4.5-7) long. Dorsal tubercles ahead of near rear margin, 33 (32-40) apart, scapular setae (sc) 12.5 (10-16), tubercles (sc) 2.5 (2.5-3) near rear margin directed (sc) divergently backwards. Coxal area with short lines; included 10 (10-11) annuli, coxal setae presented, coxae punctuaed, anterolateral setae on coxisternum $\mathbf{I}(1 b) 9.5$ (8-12) apart, 12 (11-14) long, proximal setae on coxisternum I(1a) 14 (10-15) apart, 6 (4.5-7) long, proximal setae on coxisternum II(2a) 24 (21-30) apart, $28 \mu$ (22-35) long. Prosternal apodeme present. Legs with usual series of setae. Legs I 38 (36-40), femur 9 (7-13), basiventral femoral setae (bv) 9 (8-13); genu 6 (4.5-7), antaxial genual setae (l") 25 (19-26); tibia 8 (7.5-9), paraxial tibial setae $\left(l^{\prime}\right) 5$ (4.5-6), setae located at center; tarsus 5.5 (5-7.5); tarsal empodia simple 6(5-7), 4-rayed, tarsal solenidion 5.5 (5-7.5) long knobbed, dorsal setae ( $\left.f t^{\prime \prime}\right) 13$ (10-20) long, lateral setae ( $\left.f t^{\prime}\right) 16.5$ (12-25) long. Legs II 36 (30-40) long, femur 9 (8-10) long, basiventral femoral setae (bv) 13 (1216) long; genu 6 (5-7), antaxial genual setae ( $\left(l^{\prime \prime}\right) 4.5$ (4-6); tibia 6.5 (6-7.5); tarsus 7.5 (5-9) long, solenidia 8.5 (7-10) long, knobbed, empodia 7 (5.5-8) long, (ft") 5 (4-6) long, ( $\left.f t^{\prime}\right) 23$ (20-28) long. Opisthosoma the dorsal with 27 (26-28) annuli, smooth, ventrally with 64 (61-71) annual, with round microtubercles. Setae c2 17 (13-21) long, on ventral annulus 9 (8-10) posterior to coxae; setae $d 50$ (45-60) long, on ventral annulus 25 (22-28), 38 (30-40) apart; setae e 11 (9-13) long, on ventral annulus 41 (36-44), 17.5 (14-21) apart; setae $f 25$ (22-25) long, on 6th ventral annulus from rear. Setae $h 1$ absent. Caudal setae (h2) 63 (54-70) long; Female genitalia 15.5 (14-20) long, 22 (20-27) wide, coverflap with 12 (10-12) longitudinal ridges, proximal setae on coxisternum III (3a) 8 (7-10) long, 15(13-20) apart.

MALE: Body fusiform, light yellowish in colour 179 (165-200) long, 58 (50-68) wide; light yellow. Gnathosoma length 15 (15-17), projecting obliquely downwards, pedipalp coxal setae (ep) 2 (2-3), dorsal pedipalp genual setae (d) 4 (4-5), chelicerae 13 (13-14), oral stylets 12 (12-14). Prodorsal shield 41 (38-45), long, 52 (43-65) wide; frontal lobe broad 6.5 (5-8) long, scapular setae (sc) 12.5 (10-16), tubercles (sc) 2.5 (2.5-3) near rear margin directed $(s c)$ divergently backwards. Coxal area with short lines; with 13 (12-14) annuli, and usual segments and setae present, coxae with irregular dashes. Legs with usual series of setae. Legs I 37 (30-40), femur 8 (7-11), setae (bv) 9 (8-10); genu 5 (5-6), setae (l') 22 (19-23); tibia $8(8-10)$, setae (l') 5 (4$6)$; tarsus 6 (6-8); tarsal empodia 6 (5-7), 4-rayed, tarsal solenidion 6 (5-7) long knobbed, dorsal setae ( $\left.f t^{\prime \prime}\right)$ 14(10-17) long, lateral setae (ft') 14 (12-20) long. Legs II 
33 (31-40) long, femur 8 (8-10) long, setae (bv) 13 (12-15) long; genu 5 (5-6), setae $\left(l^{\prime \prime}\right) 4.5$ (4-6); tibia 6.5 (6-7.5); tarsus 7 (5-8) long, solenidia 7 (7-9) long, knobbed, empodia 6.5 (6-7.5) long, ( $\left.f t^{\prime \prime}\right) 5$ (4-6) long, ( $\left.f t^{\prime}\right) 20$ (18-23) long. Opisthosoma: dorsal side with 27 (26-31) annuli, ventrally with 58 (55-59) microtuberculate annuli. Setae $c 211$ (10-13) long, on ventral annulus 10 (9-10); setae $d 40$ (38-45) long on ventral annulus 23 (22-27); setae e 13 (12-13) on ventral annulus 40 (39-46). Setae $f 24$ (23-26) on 6 (5-6) ventral annulus from rear. Caudal setae (h2) 60 (50-62) long. Male genitalia 12.5 (12-14) long, 14 (12-16) wide, proximal setae on coxisternum III (3a) 7.5 (7-8) long.

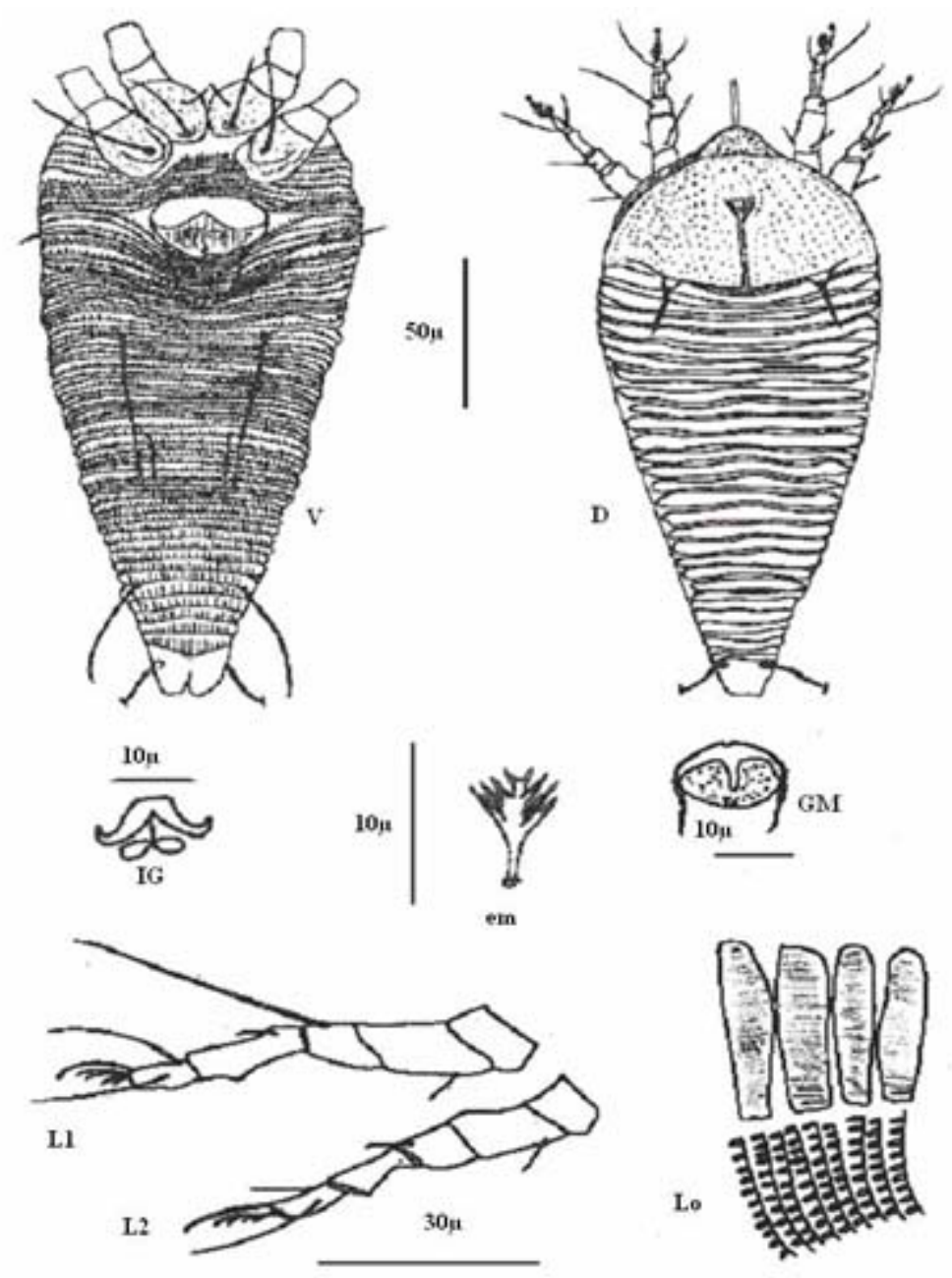

Fig. 1: Tegolophus guavae (Boczek, 1960) - D, dorsal view of mite; V, ventral view; IG, internal genitalia of female; em, empidium; GM, genital region male; L1, L2, legs I,II; Lo lateral opisthosoma.

Relation to host - Vagrant on lower and upper surface of leaves causing rust on leaves.

Aceria ziziphi (Mohanasundaram, 1990) (Fig. 2)

Aceria ziziphi Mohanasundaram, 1990

Material examined: Qalubia; 19.V.2012, (Ashraf EL-Halawany), Ziziphus spinachristi Willd., (7웅, 5ふ⿱人) $\left(30^{\circ} 15^{\prime} 50.46^{\prime \prime N}\right.$; 31 1 14'51.85"E).

FEMALE: Body worm-like, white to white yellowish in colour 215 (207-230) long, 50 (45-60) wide. Gnathosoma 22 (19-25), projecting evenly downwards, pedipalp 
coxal setae (ep) 4 (3-5), dorsal pedipalp prodorsal shield rounded shape 25 (24-26) long, 34 (30-35) wide; median shield line present at curved and touching the admedians at the posterior end; the second submedians curved and genual setae (d) 3 (3-4), chelicerae 20 (17-22), oral stylets 10 (9-10). branched, basal half, admedians complete, sinuate, first submedian represented in anterior half of the shield, sides of shield with short lines and granular. Dorsal tubercles at shield margin, projecting setae backward; dorsal tubercles 23 (20-25) apart, scapular setae (sc) 26 (20-30), tubercles (sc) 2.5 (2-3). Coxal area with 6 annuli, usual segments and setae present, coxae punctuation, broadly joined first, anterolateral setae on coxisternum I (1b) 8 (7-9) apart, 9 (8-10) long, proximal setae on coxisternum I(1a) 8 (7-9) apart, 22 (19-25) long, proximal setae on coxisternum II(2a) 27 (25-28) apart, $30 \mu$ (29-31) long. Legs with usual series of setae. Legs I 35 (33-40), femur 9 (7-10), basiventral femoral setae (bv) 11 (10-13); genu 6 (5-7), antaxial genual setae (l") 27 (25-30); tibia 8 (7-8.5), paraxial tibial setae $\left(l^{\prime}\right) 5(4-5)$, setae located at a basal one third; tarsus 6 (5-7); tarsal empodia simple 8 (7-9), 7-rayed, tarsal solenidion 9 (8-11) long slightly curved un knobbed, dorsal setae ( $\left.f t^{\prime \prime}\right) 5$ (4-5.5) long, lateral setae ( $\left.f t^{\prime}\right) 20$ (19-23) long, tarsal seta (u') 5 (4-5). Legs II 32 (27-35) long, femur 9 (7-10) long, basiventral femoral setae (bv) 10 (9-11) long; genu 5 (4.5-6), antaxial genual setae (l") 9 (7-10); tibia 7 (6-7.5); tarsus 8 (7-8) long, solenidia 10 (9-12) long, unknobbed, empodia 8 (7-9) long, (ft") 6 (5-7) long, (ft') 24 (20-25) long. Opisthosoma with 65 (62-70) annuli, with round microtuberculate, microtubercles elongated in about 6 rings on tergites behind shield, rest of microtubercles dot-like last six sternites with microstriae. Setae $c 225$ (22-28) long, 50 (47-60) apart, on ventral annulus 10 (10-11) posterior to coxae; setae $d 70$ (60-75) long, on ventral annulus 21 (21-23), 45 (40-50) apart; setae e 9 (8-10) long, on ventral annulus 37 (36-38), 25 (25-50) apart; setae $f 27$ (23-30) long, on $6^{\text {th }}$ ventral annulus from rear. Accessory setae h1 4 (4-5), Caudal setae (h2) 95 (85-100) long; Female genitalia 15 (13-18) long, 19 (17-22) wide, coverflap with 12 (10-12) longitudinal ridges, proximal setae on coxisternum III (3a) 35 (32-40) long, 15 (1117) apart.

MALE: Body worm-like, 165 (145-173) long, 46 (43-50) thick, white. Gnathosoma 20 (17-23), evenly down, pedipalp coxal setae (ep) 4 (3-5), dorsal pedipalp genual setae (d) 3 (3-4), chelicerae 19 (17-22), oral stylets 10 (9-10). Prodorsal shield rounded 27 (25-32) long, 32 (30-33) wide; median shield line present at basal half, admedians complete, sinuate, first submedian represented in anterior half of the shield, curved and touching the admedians at the posterior end; the second submedians curved and branched. Sides of shield with short lines and granular. Dorsal tubercles at shield margin, projecting setae backwards; dorsal tubercles 22 (19-23) apart, scapular setae (sc) 27 (25-28), tubercles (sc) 2.5 (2-3). Coxal area with 6 annuli, and usual segments and setae present coxae punctuation, broadly joined first, anterolateral setae on coxisternum I (1b) 9(7-10) apart, 8 (7-10) long, proximal setae on coxisternum I(1a) 8(7-8) apart, 18 (17-20) long, proximal setae on coxisternum II(2a) 20 (17-22) apart, 32 (27-35) long. Legs with usual series of setae. Legs I 32 (27-35), femur 8 (7-9), basiventral femoral setae (bv) 10 (8-12); genu 5 (4-5), antaxial genual setae $\left(l^{\prime \prime}\right) 19$ (18-20); tibia 7 (6-7), paraxial tibial setae $\left(l^{\prime}\right) 9(8-10)$, setae located at a basal one third; tarsus 5 (5-6); tarsal empodia simple 7 (7-8), 7-rayed, tarsal solenidion 10 (9-12) long slightly curved unknobbed, dorsal setae (ft") 5 (4-5) long, lateral setae $\left(f t^{\prime}\right) 20$ (18-25) long, tarsal setae $\left(u^{\prime}\right) 5$ (4-5). Legs II 27 (25-30) long, femur 8 (8-10) long, basiventral femoral setae (bv) 10 (10-11) long; genu 5 (4.55.5), antaxial genual setae ( $\left(l^{\prime \prime}\right) 11$ (11-12); tibia 7 (6-8); tarsus 5 (5-6) long, solenidia 10 (9-12) long, empodia 7 (6-8) long, (ft") 7 (7-8) long, (ft') 16 (15-17) long. 
Opisthosoma with 62 (60-66) annuli, with round microtuberculate, microtubercles elongated in about 6 rings on tergites behind shield. Setae $c 220$ (16-21) long, 40 (3846) apart, on ventral annulus 10 (10-11) posterior to coxae; setae $d 38$ (31-42) long, on ventral annulus 20 (20-21); setae e 5 (5-6) long, on ventral annulus 35 (34-36); setae $f 20$ (16-24) long, on $6^{\text {th }}$ ventral annulus from rear. Accessory setae $h 16$ (5-7), Caudal setae (h2) 68 (65-70) long; male genitalia 11 (10-11.5) long, 14 (13-15) wide, setae (3a) 10 (9-12) long, 9.5 (9-11) apart.

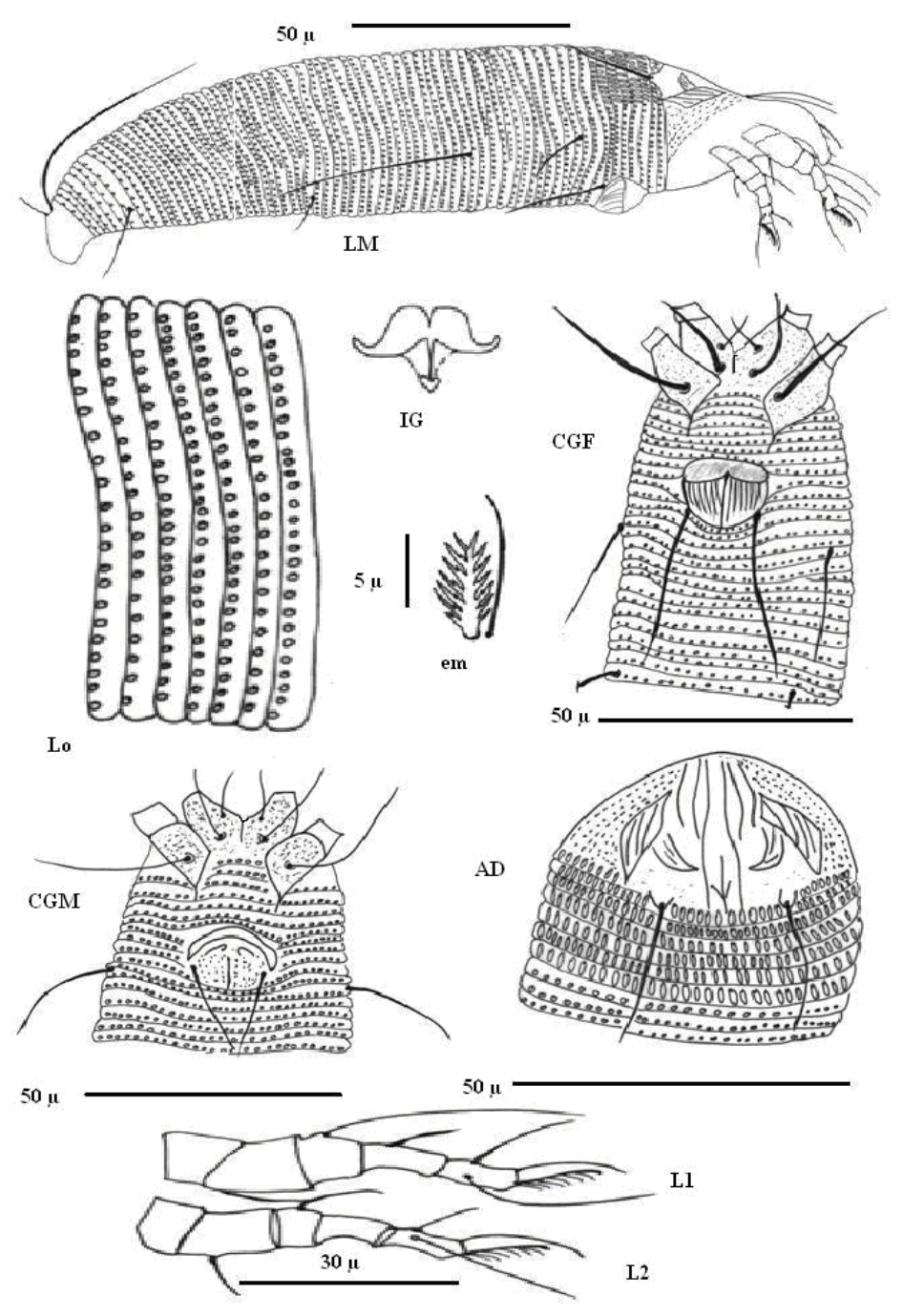

Fig. 2: Aceria ziziphi (Mohanasundaram, 1990) - LM, lateral view of mite; CGF, coxi-genital region of female; IG, internal genitalia of female; em, empidium; CGM, genital region of male; L1, L2, legs I,II; Lo lateral view of opisthosoma; AD, Anterior-dorsal view of mite.

Relation to host -This species was found in the tender apical shoots between hairs; causes no damage.

\section{Checklist of Eriophyid mites}

Although the diversity of Egyptian agro-ecosystem, the total number of eriophyid mites in Egypt does not exceed $1.5 \%$ of the recorded number in the world. The collected references on the eriophyid mites in Egypt recorded 65 species during the period from 1919 up to date. The collective work on eriophyid mites in Egypt was conducted in 1984 and 47 species have been recorded in this work. These species included all eriophyid mite species which recorded before 1984 except four species, 
namely: Aceria alfierii Sayed, Aceria arabicae Meyer, Cecidophyes violae (Nalepa) and Oxycenus maxwelli (Keifer). On the other hand, 12 species have been recorded during the period after 1984 up to date. Table (1) includes 65 eriophyoid mite species in super family Eriophyoidea reported from Egypt. These species belong to three families, seven sub families, seven tribes and 27 genera. Eriophyidae is the largest family represented by 24 genera and 63 species. Family Phytoptidae contains two genera and two species. Diptilomiopidae contains one genus and one species.

The recorded species belong to the genera: Abacarus (2), Aceria (25), Aculops (3), Aculus (4), Calepitrimerus (1), Cecidophyes (1), Colomerus (2), Dicruvasates (1), Diptilomiopus (1), Epitrimerus (1), Eriophyes (3), Heterotergum (1), Mackiella (1), Metaculus (1), Neooxycenus (1), Neotegonotus (1), Oxycenus (2), Oziella (1), Phyllocoptes (2), Phyllocoptruta (2), Retracrus (1), Rhyncaphytoptus (1), Tegolophus (3), Tegonotus (1), Tetra (1), Vasates (1) and Vittacus (1).

The eriophyid mite species, their host plant and references in Egypt were listed in table (2).

Table 2: List of eriophyoid mites recorded from Egypt up to date.

\begin{tabular}{|c|c|c|c|}
\hline Classification & Scientific name & Host plant & Reference \\
\hline $\begin{array}{l}\text { Family: Diptilomiopidae } \\
\text { Subfamily: } \\
\text { Diptilomiopinae }\end{array}$ & Diptilomiopus ficus Attiah, 1967 & Ficus carica L., (Moraceae) & Attiah, 1967 \\
\hline \begin{tabular}{|l|} 
Subfamily: \\
Rhyncaphytoptinae
\end{tabular} & Rhyncaphytoptus ficifoliae Keifer, 1939a & Ficus carica L., (Moraceae) & Zaher et al., 1978 \\
\hline \multirow[t]{3}{*}{$\begin{array}{l}\text { Family: Eriophyidae } \\
\text { Subfamily: } \\
\text { Cecidophyinae } \\
\text { Tribe: Cecidophyini }\end{array}$} & Cecidophyes violae (Nalepa, 1902) & $\begin{array}{l}\text { Viola riviniana Eichenb., } \\
\text { (Violaceae) }\end{array}$ & Osman \& Zohdy, 1976 \\
\hline & Colomerus oculivitis (Attiah, 1970) & Vitis vinifera L., (Vitaceae) & Attiah, 1970 \\
\hline & Colomerus vitis (Pagenstecher, 1857) & Vitis vinifera L., (Vitaceae) & Zaher et al., 1978 \\
\hline \multirow[t]{14}{*}{$\begin{array}{l}\text { Subfamily: Eriophyinae } \\
\text { Tribe: Aceriini }\end{array}$} & $\begin{array}{l}\text { Aceria aegyptiacus (Soliman \& Abou-Awad, } \\
\text { 1977) }\end{array}$ & $\begin{array}{l}\text { Allium sativum, (Liliaceae, } \\
\text { Alliaceae) }\end{array}$ & $\begin{array}{l}\text { Soliman \& Abou-Awad, } \\
1977\end{array}$ \\
\hline & $\begin{array}{l}\text { Aceria aegypticus (Rasmy \& Abou-Awad, } \\
\text { 1978) }\end{array}$ & \begin{tabular}{|l|}
$\begin{array}{l}\text { Marrubium alysson L., } \\
\text { (Lamiaceae) }\end{array}$ \\
\end{tabular} & Rasmy\& Abou- Awad, 1978 \\
\hline & Aceria alfierii Sayed, 1946 & $\begin{array}{l}\text { Pluchea dioscoridis (L.), } \\
\text { (Asteraceae) }\end{array}$ & Sayed, 1946b \\
\hline & Aceria arabicae Meyer, 1990 & Acacia nilotica (L.), (Fabaceae) & Sayed, 1946b \\
\hline & $\begin{array}{l}\text { Aceria benghalensis (Soliman \& Abou- } \\
\text { Awad, 1977) }\end{array}$ & $\begin{array}{l}\text { Ficus benghalensis L., } \\
\text { (Moraceae) }\end{array}$ & $\begin{array}{l}\text { Soliman \& Abou-Awad, } \\
1977\end{array}$ \\
\hline & Aceria cynodoniensis (Sayed, 1946) & $\begin{array}{l}\text { Cynodon dactylon (L.) Pers., } \\
\text { (Poaceae) }\end{array}$ & Sayed, 1946a \\
\hline & $\begin{array}{l}\text { Aceria daturae (Soliman \& Abou-Awad, } \\
\text { 1977) }\end{array}$ & $\begin{array}{l}\text { Datura stramonium L., } \\
\text { (Solanaceae) }\end{array}$ & $\begin{array}{l}\text { Soliman \& Abou-Awad, } \\
1977\end{array}$ \\
\hline & Aceria dioicae (Keifer, 1979) & $\begin{array}{l}\text { Tamarix dioica Roxb., } \\
\text { (Tamaricaceae) }\end{array}$ & $\begin{array}{l}\text { Abou-Awad and El- } \\
\text { Borolossy, } 1995\end{array}$ \\
\hline & $\begin{array}{l}\text { Aceria dioscoridis (Soliman \& Abou-Awad, } \\
\text { 1977) }\end{array}$ & $\begin{array}{l}\text { Pluchea dioscoridis L., } \\
\text { (Asteraceae) }\end{array}$ & $\begin{array}{l}\text { Soliman \& Abou-Awad, } \\
1977\end{array}$ \\
\hline & Aceria eriobotryae (Keifer, 1938a) & $\begin{array}{l}\text { Eriobotrya japonica (Thunb.) } \\
\text { Lindl., (Rosaceae) }\end{array}$ & Zaher et al., 1978 \\
\hline & Aceria ficus (Cotté, 1920) & Ficus carica L., (Moraceae) & Hassan, 1934 \\
\hline & $\begin{array}{l}\text { Aceria imperata (Zaher \& Abou-Awad, } \\
\text { 1978) }\end{array}$ & $\begin{array}{l}\text { Imperata cylindrica (L.) Beauv., } \\
\text { (Poaceae) }\end{array}$ & Zaher \& Abou-Awad, 1978 \\
\hline & Aceria kenyae (Keifer, 1966) & $\begin{array}{l}\text { Mangifera indica L., } \\
\text { (Anacardiaceae) }\end{array}$ & Zaher, 1984 \\
\hline & Aceria lycopersici (Wolffenstein, 1879) & $\begin{array}{l}\text { Solanum lycopersicum L. } \\
\text { (Solanaceae) }\end{array}$ & Hassan, 1934 \\
\hline
\end{tabular}


Table (2) (continued)

\begin{tabular}{|c|c|c|c|}
\hline Classification & Scientific name & Host plant & Reference \\
\hline & Aceria mangiferae (Sayed, 1946) & $\begin{array}{l}\text { Mangifera indica L., } \\
\text { (Anacardiaceae) }\end{array}$ & Sayed, 1946a \\
\hline & Aceria melongena (Zaher \& Abou-Awad, 1978) & $\begin{array}{l}\text { Solanum melongena L., } \\
\text { (Solanaceae) }\end{array}$ & $\begin{array}{l}\text { Zaher \& Abou- } \\
\text { Awad, } 1978\end{array}$ \\
\hline & Aceria mori (Keifer, 1939a) & Morus alba L., (Moraceae) & $\begin{array}{l}\text { Zaher \& Abou- } \\
\text { Awad, 1980a. }\end{array}$ \\
\hline & Aceria neocynarae (Keifer, 1939b) & $\begin{array}{l}\text { Cynara scolymus L., } \\
\text { (Asteraceae) }\end{array}$ & $\begin{array}{l}\text { Zaher \& Abou- } \\
\text { Awad, 1980a }\end{array}$ \\
\hline & Aceria nilotica (Abou-Awad \& Nasr, 1983a) & $\begin{array}{l}\text { Cynodon dactylon (L.) Pers., } \\
\text { (Poaceae) }\end{array}$ & $\begin{array}{l}\text { Abou-Awad \& Nasr, } \\
\text { 1983a }\end{array}$ \\
\hline & Aceria oleae (Nalepa, 1900) & $\begin{array}{l}\text { Olea europaea L., } \\
\text { (Oleaceae) }\end{array}$ & $\begin{array}{l}\text { Zaher \& Abou-Awad, } \\
\text { 1980b }\end{array}$ \\
\hline & Aceria olivi (Zaher \& Abou-Awad, 1979) & $\begin{array}{l}\text { Olea europaea L., } \\
\text { (Oleaceae) }\end{array}$ & $\begin{array}{l}\text { Zaher \& Abou- } \\
\text { Awad, } 1979\end{array}$ \\
\hline & Aceria sheldoni (Ewing,1937) & Citrus spp., (Rutaceae) & Zaher et al., 1978 \\
\hline & Aceria sycamori (Soliman \& Abou-Awad, 1977) & $\begin{array}{l}\text { Ficus sycomorus L., } \\
\text { (Moraceae) }\end{array}$ & $\begin{array}{l}\text { Soliman \& Abou- } \\
\text { Awad, } 1977\end{array}$ \\
\hline & Aceria tulipae (Keifer, 1938a) & $\begin{array}{l}\text { Allium sativum L., (Liliaceae, } \\
\text { Alliaceae) }\end{array}$ & Zaher et al., 1978 \\
\hline & Aceria ziziphi Mohanasundaram, 1990 & $\begin{array}{l}\text { Ziziphus spina-christi Willd } \\
\text { (Rhamnaceae) }\end{array}$ & New record \\
\hline \multirow[t]{3}{*}{ Tribe: Eriophyini } & Eriophyes acanthus Osman \& Abo-Taka, 1989 & $\begin{array}{l}\text { Eranthemum pulchellum } \\
\text { Andr., (Acanthaceae) }\end{array}$ & $\begin{array}{l}\text { Osman \& Abo-Taka, } \\
1989\end{array}$ \\
\hline & Eriophyes nalepi (Zaher \& Abou-Awad, 1978) & $\begin{array}{l}\text { Pyrus communis L., } \\
\text { (Rosaceae) }\end{array}$ & $\begin{array}{l}\text { Zaher \& Abou- } \\
\text { Awad, } 1978\end{array}$ \\
\hline & Eriophyes pyri (Pagenstecher, 1857) & $\begin{array}{l}\text { Pyrus communis L., } \\
\text { (Rosaceae) }\end{array}$ & Hassan, 1934 \\
\hline \multirow[t]{8}{*}{$\begin{array}{l}\text { Subfamily: Phyllocoptinae } \\
\text { Tribe: Anthocoptini }\end{array}$} & $\begin{array}{l}\text { Abacarus cynodonsis Abou-Awad \& Nasr, } \\
\text { 1983a }\end{array}$ & $\begin{array}{l}\text { Elymus repens (L.) (Poaceae, } \\
\text { Cyperaceae) }\end{array}$ & $\begin{array}{l}\text { Abou-Awad \& Nasr, } \\
\text { 1983a }\end{array}$ \\
\hline & Abacarus hystrix (Nalepa, 1896) & $\begin{array}{l}\text { Elymus repens (L.) (Poaceae, } \\
\text { Cyperaceae) }\end{array}$ & Zaher, 1984 \\
\hline & Aculops acaciae Abou-Awad \& Elsawi, 1993 & $\begin{array}{l}\text { Acacia nilotica (L.), } \\
\text { (Fabaceae) }\end{array}$ & $\begin{array}{l}\text { Abou-Awad \& } \\
\text { Elsawi, } 1993 \\
\end{array}$ \\
\hline & Aculops lycopersici (Tryon, 1917) & $\begin{array}{l}\text { Solanum lycopersicum L. } \\
\text { (Solanaceae) }\end{array}$ & Zaher et al., 1978 \\
\hline & Aculops nilotica Abou-Awad, 1979a & $\begin{array}{l}\text { Mentha spicata L., } \\
\text { (Lamiaceae) }\end{array}$ & Abou-Awad, 1979a \\
\hline & Aculus fockeui (Nalepa \& Trouessart, 1891) & $\begin{array}{l}\text { Prunus domestica L., } \\
\text { (Rosaceae) }\end{array}$ & Zaher et al., 1978 \\
\hline & Aculus malus (Zaher \& Abou-Awad, 1979) & $\begin{array}{l}\text { Malus domestica Borkh., } \\
\text { (Rosaceae) }\end{array}$ & $\begin{array}{l}\text { Zaher \& Abou- } \\
\text { Awad, } 1979\end{array}$ \\
\hline & Aculus schlechtendali (Nalepa, 1890) & $\begin{array}{l}\text { Malus domestica Borkh., } \\
\text { (Rosaceae) }\end{array}$ & $\begin{array}{l}\text { Zaher \& Abou- } \\
\text { Awad, 1980a }\end{array}$ \\
\hline
\end{tabular}


Table (2) (continued)

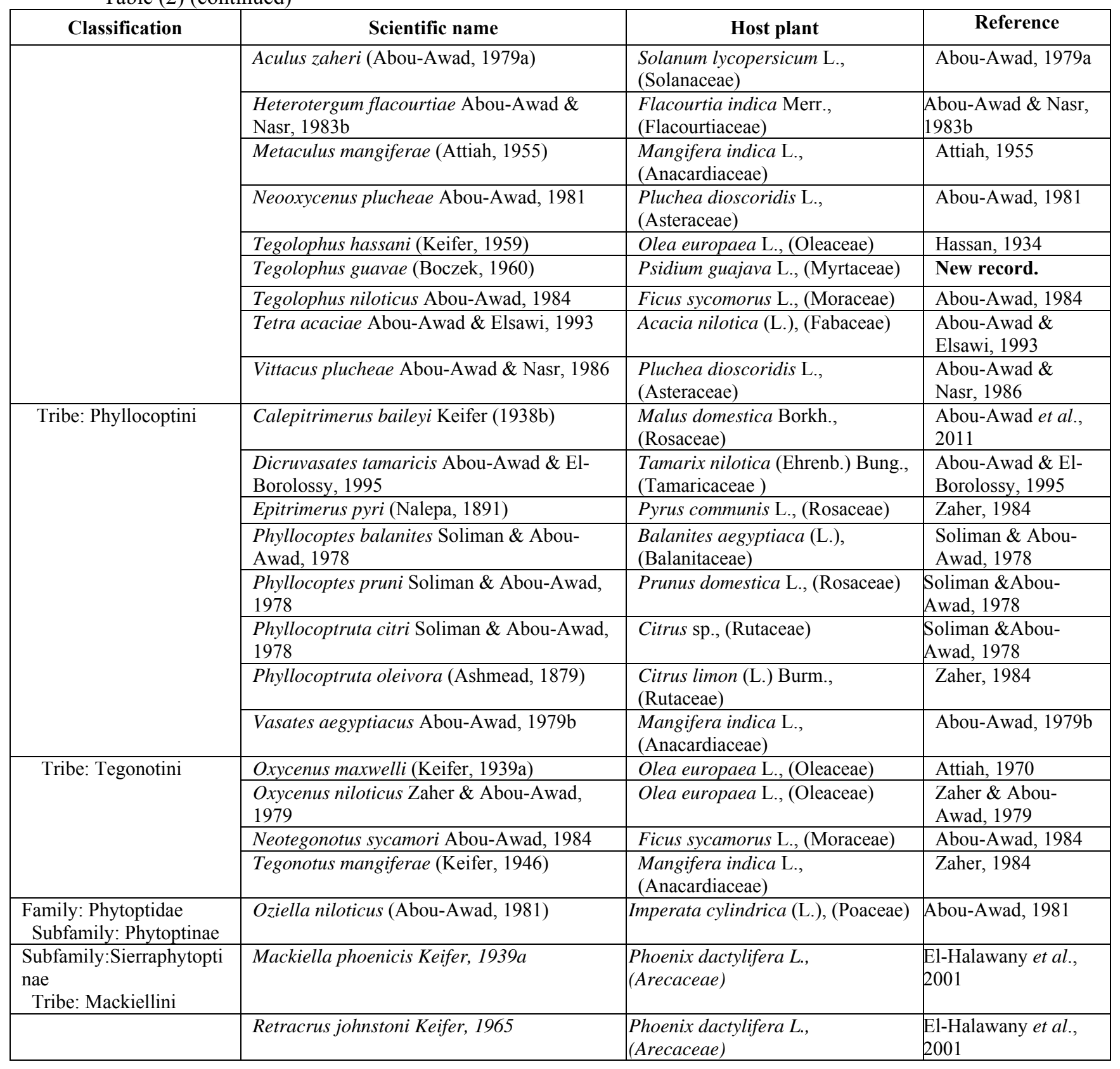

\section{ACKNOWLEDGEMENTS}

The author is grateful to many people who helped in various stages of this study, especially Professor Mohamed A. Zaher (Faculty of Agric. Cairo Univ.) and Prof. Badawi A. Abou-Awad (National Research Centre, Dokki, Cairo). Also, he would like to thank Dr. Pratibha Menon (Indian Agricultural Research Institute, New Delhi, India) and undoubtedly Dr. Jan Boczek (Agric. Univ. of Warsaw, Poland) for his very valuable suggestions to the author to erect the new record species. The author is grateful to Prof. M. Abou-Setta and Dr. Alaa Halawa for reviewing an earlier draft of this paper. 


\section{REFERENCES}

Abou-Awad, B.A. (1979a): Two new species of genus Aculops in Egypt (Eriophyoidea: Eriophyidae). Acarologia, 21(2): 234-238.

Abou-Awad, B.A. (1979b): New species of genus Vasates in Egypt (Acari: Eriophyoidea: Eriophyidae). Acarologia, 21(3-4): 389-391.

Abou-Awad, B.A. (1981): Some eriophyoid mites from Egypt with descriptions of two new species (Acari: Eriophyoidea). Acarologia, 22(4): 367-372.

Abou-Awad, B.A. (1984): Two new eriophyid species infesting sycamore trees in Egypt (Acari: Eriophyoidea: Eriophyidae). Acarologia, 25(1): 21-25.

Abou-Awad, B.A. and El-Borolossy, M.A. (1995): Two eriophyid mites on tamarisk trees in Egypt (Acari: Eriophyoidea: Eriophyidae). Acarologia, 36(2): 145148.

Abou-Awad, B.A. and Elsawi, S.A. (1993): Two new species of eriophyid mites injurious to Acacia trees from Egypt (Acari, Eriophyidae). Deutsche. Entomol. Z., 40(2): 403-406.

Abou-Awad, B.A. and Nasr, A.K. (1983a): Occurrence of the eriophyid mites as new pests of Bermuda grass, Cynodon dactylon (L.) Pers. in Egypt. Internat. J. Acarol., 9(4): 183-187.

Abou-Awad, B.A. and Nasr, A.K. (1983b): Heterotergum flacourtiae n. sp. from Egypt (Acari: Eriophyidae). Internat. J. Acarol., 9(4): 179-181.

Abou-Awad B.A., Nasr A.K. (1986): An eriophyd mite, Vittacus plucheae sp. n. (Acari: Eriophyoidea: Eriophyidae), onPluchea dioscoridis L. (Compositae) from Egypt. Acarologia, 27(2): 159-161.

Abou-Awad, B.A., Afia, S.I. and Al-Azzazy, M.M. (2011): The life-history and biomics of the apple rust mite, Calepitrimerus baileyi (Acari: Eriophyidae). Acarines, 5(1) 57-63.

Amrine, J.W.Jr. and Stasny, T.A. (1996): Corrections to the catalog of the Eriophyoidea (Acarina: Prostigmata) of the world. Internat. J. Acarol., 22(4): 295-304.

Amrine, J.W. Jr.; Stasny, T.A. and Flechtmann, C.H.W. (2003): Revised keys to world genera of Eriophyoidea (Acari: Prostigmata). Indira Publishing House, West Bloomfield, MI. 244 pp.

Ashmead, W.H. (1879): Injurious and beneficial insects found on the orange trees in Florida. Can. Entomol., 11(8): 159-160.

Attiah, H.H. (1955): A new eriophyid mite on mango from Egypt (Acarina). Bull. Soc. Ent. Ėgypte, 39: 379-383.

Attiah, H.H. (1967): Two new species of mites on figs from Egypt (Acarina). Bull. Soc. Ent. Égypte, 51(1): 1-5.

Attiah, H.H. (1970): New records of eriophyid mites from Egypt. (Acarina). Bull. Soc. Ent. Égypte, 54: 43-47.

Boczek, J. (1960): A new genus and three new species of eriophyid mites (Acarina). J. Kans. Entomol. Soc., 33(1): 9-14.

Cotté, J. (1920): Deux parasites de la figue sauvage. Bull. Soc. Pathol. Veg. France, 7 : 26-30.

De Lillo, E. and Amrine, J.W (2009): Catalogue of the Eriophyoidea of the world. Version computer of Filemaker Pro 4.0.

Dçbski, B. (1919): Nouvelles additions à ma liste des cédicies d'Égypte. Bull. Soc. Fouad $1^{\text {er }}$ Entomol., 3: 65-70. 
El-Halawany, M.E., Abdel-Samad, M.A. and El-Naggar, M.E. (2001): Mites inhabiting date palms. In Second Inter. Conf. on Date Palms (Al-Ain, UAE, March 25-27, 2001) pp.366-373.

Ewing, H.E. (1937): A new eriophyid mite from lemon trees (Acarina: Eriophyidae). Proc. Entomol. Soc. Wash., 29: 193-194.

Hassan, A.S. (1934): Notes on the Eriophyidae of Egypt. Bull. Soc. Fouad $1^{\text {er }}$ Entomol., 18: 440-444.

Keifer, H.H. (1938a): Eriophyid studies I. Bull. Calif. Dept. Agr. 27: 181-206.

Keifer, H.H. (1938b): Eriophyid studies II. Bull. Calif. Dept. Agr., 27: 301-323.

Keifer, H.H. (1939a): Eriophyid studies III. Bull. Calif. Dept. Agr., 28: 144-162.

Keifer, H.H. (1939b): Eriophyid studies V. Bull. Calif. Dept. Agr., 28: 328-345.

Keifer, H.H. (1939c): Eriophyid studies VI. Bull. Calif. Dept. Agr., 28: 416-426.

Keifer, H.H. (1946): Eriophyid studies XVI. Bull. Calif. Dept. Agr., 35: 39-48.

Keifer, H.H. (1959): Eriophyid studies XXVII. Occasional Papers. Calif. Dept. Agr., 1: $1-18$.

Keifer, H.H. (1965): Eriophyid studies B-16. Bur. Ent., Calif. Dept. Agric.: 1-20.

Keifer, H.H. (1966): Eriophyid studies B-18. Bur. Ent., Calif. Dept. Agric.: 1-20.

Keifer, H.H. (1979): Eriophyid studies C-16. ARS-USDA: 1-24.

Lindquist, E.E. (1996): External anatomy and notation of structures, pp. 3-31. In: Lindquist, E. E., M. W. Sabelis and J. Bruin [Eds.]. Eriophyoid Mites: Their Biology, Natural Enemies and Control. Elsevier, Amsterdam, The Netherlands.

Meyer, S.M.K. (1990): A review of species of Aceria Keifer (Acari: Eriophyidae) associated with Acacia spp. Internat. J. Acarol., 16(3): 149-173.

Mohanasundaram, M. (1990): Studies on the genus Aceria (Acari: Eriophyidae) from South India. Indian J. Acarol. 12(1\&2): 15-88.

Nalepa, A. (1890): Zur Systematik der Gallmilben. Sitzb. kaiser. Akad. Wiss. Math.Nat., Wien, 99(2): 40-69.

Nalepa, A. (1891): Genera und Species der Familie Phytoptida. Denkschr. kaiser. Akad. Wiss. Math.-Nat., Wien, 58: 867-884.

Nalepa, A. (1896): Neue Gallmilben. Anz. kais. Akad. Wiss., Math.-Nat. Kl., Wien, 33(10): 108-110.

Nalepa, A. (1900): Neue Gallmilben. Anz. kais. Akad. Wiss., Math.-Nat, K1., Wien, 37(15): 154-156.

Nalepa, A. (1902): Neue Gallmilben. Anz. kais. Akad. Wiss., Math.-Nat. Kl., Wien, 39(26): 335-336.

Nalepa, A. and Trouessart E. (1891): Diagnoses d'acariens nouveaux Le Naturaliste. Revue Illustrée des Sciences Naturelles Sér. 2, 13(93): 25-26.

Osman, A.A. and Abo-Taka S.M. (1989): Eriophyes acanthus n. sp. from Egypt (Acari: Eriophyoidea: Eriophyidae). Internat. J. Acarol., 15(1): 53-54.

Osman, A.A. and Zohdy G.I. (1976): A new record of the eriophyid mite Phyllocoptes violae (Nal.) on violet plant in Egypt (Acari: Eriophyidae). Bull. Soc. Ent. Égypte, 60: 319-321.

Pagenstecher, H. A. (1857): Über Milben besonders die Gattung Phytoptus. Verh. Naturhist. Med. Ver., Heidelberg, 1(2) (Bund 1857-1859): 46-53.

Rasmy, A.H. and Abou-Awad B.A. (1978): A new species of genus Eriophyes from Egypt (Acarina: Eriophyoidea: Eriophydae). Acarologia, 20(3): 385-387.

Sayed, M.T. (1946a): Aceria mangiferae nov. spec. Bull. Soc. Fouad $1^{\text {er }}$ Entomol., 30: $7-10$. 
Sayed, M.T. (1946b): Three new eriophid mites from Egypt (Acarina, Eriophyidae). Bull. Soc. Fouad $1^{\text {er }}$ Entomol., 30: 149-154.

Soliman, Z.R. and Abou-Awad, A. (1977): Five new species of the genus Eriophyes in the A.R.E. (Acarina: Eriophyoidea: Eriophyidae). Acarologia, 19(4): 668-677.

Soliman, Z.R. and Abou-Awad A. (1978): A new species of the genus Phyllocoptruta in the A.R.E. (Acarina: Eriophyoidea: Eriophyidae). Acarologia, 20(1): $109-111$.

Tryon, H. (1917): Report of the Entomologist and Vegetable Pathologist. Queensland Dept. Agric. \& Stock Rept., 1916/1917: 49-63.

Wolffenstein, O. (1879): Phytoptus lycopersici W. Monatsschrif des Vereins zur Befoerderung des Gartenbaues in den Konigl. Pruessischen Staaten, 22: 424426.

Zaher, M.A. (1984): Survey and ecological studies on phytophagous, predaceous and soil mites in Egypt. I: Phytophagous mites in Egypt (Nile valley and Delta). PI 480 Programme. USA Project No. EG. ARS, 30. Grant. No, FG, Eg., 81. 228pp.

Zaher M.A., Abou-Awad B.A. 1979. Three new species of the genera Eriophyes and Phytoptus in Egypt. (Eriophyoidea: Eriophyidae). Acarologia 20(4): 556-562.

Zaher, M.A. and Abou-Awad B.A. (1979): Three new species of the genera Eriophyes and Phytoptus in Egypt. (Eriophyoidea: Eriophyidae). Acarologia, 20(4): 556-562.

Zaher, M.A. and Abou-Awad, B.A. (1980a): A new species and new record of some eriophyid mites in Egypt (Eriophyoidea: Eriophyidae). Acarologia, 21(1): 61-64.

Zaher, M.A. and Abou-Awad, B.A. (1980b): Two new eriophyid species infesting olive trees in Egypt (Eriophyoidea: Eriophyidae). Acarologia, 21(1): 65-69.

Zaher, M.A., Solima, Z.R., Rasmy, A.H. and Abou-Awad, B.A. (1978): Eriophyoid mites of Egypt. $4^{\text {th }}$ Conf. Control. NRC. Cairo: 815-817.

\section{ARABIC SUMMARY}

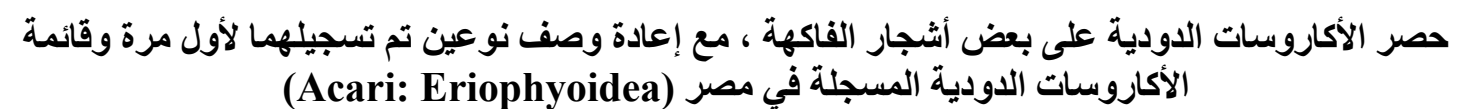

$$
\text { قسم بحوث أكاروس الفاكهةـ معهد بحوث وقاية النباتاتـ مركز الثلوف البحوث الزر اعيةـ الدقي ـ الجيزةـ مصر }
$$

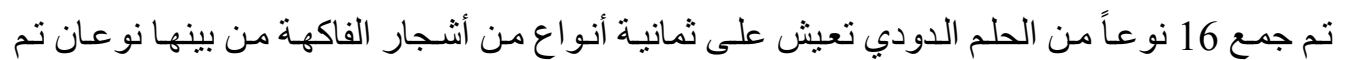

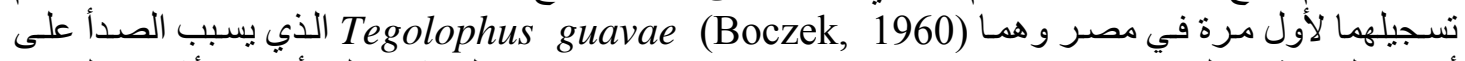

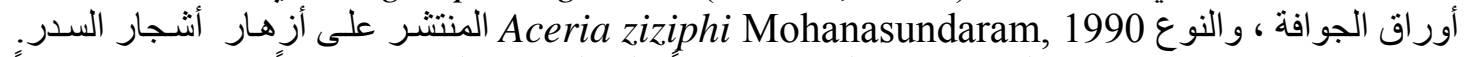

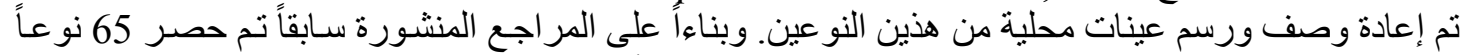

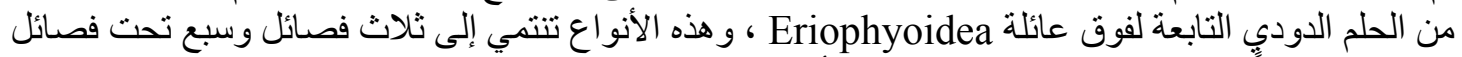
يتبعها 27 جنساً ، وتم عمل قائمة مرجعية لهذه الأنو اع. 